\title{
EQUATIONS OF MOTION FOR A CHARGED PARTICLE IN $n$-DIMENSIONAL MAGNETIC FIELD
}

\author{
W. Florek AND M. Thomas \\ Institute of Physics, Adam Mickiewicz University \\ Umultowska 85, 61-614 Poznań, Poland
}

\begin{abstract}
The equation of motion for a charged particle moving in the $n$-dimensional constant magnetic field is obtained for any linear gauge and any metric tensor by generalization of Johnson and Lipmann's approach. It allows to consider the magnetic orbits in the $n$-dimensional space. It is shown that the movement of a particle can always be decomposed into a number of two-dimensional cyclotronic motions and a free particle part.
\end{abstract}

PACS numbers: $02.20 .-\mathrm{a}, 03.65 . \mathrm{Bz}$

\section{Introduction}

The motion of a charged particle in a constant magnetic field has been considered by many authors since pioneering works of Landau and Peierls [1]. Johnson and Lipmann [2] in their wide-cited paper treated this problem in both relativistic and nonrelativistic quantum approach, introducing in fact operators for coordinates of the orbit center. Another important series of papers was started by independent, but very closely related, works of Brown [3] and Zak [4] concerning a Bloch electron in a magnetic field.

This paper is inspired by a comment of Menon and Agrawal [5] in which the $n$-dimensional magnetic field was determined. We consider a general form of the $n$-dimensional vector potential $\boldsymbol{A}$ determining a constant and uniform magnetic field $\boldsymbol{H}$. The equations of motion, analogous to those given by Johnson and Lipmann [2], are obtained and they lead to decomposition of the coordinates into two parts corresponding to the cyclotron and free movements, respectively. In the first part, the movement of the orbit center and the relative coordinates can be distinguished.

\section{Linear gauge and equations of motion in general form}

The $n$-dimensional magnetic field can be defined as the antisymmetrization of $\nabla \boldsymbol{A}$, with $\nabla$ denoting the $n$-dimensional gradient operator, i.e. [5] 


$$
\mathrm{H}_{j k}=\partial_{j} A_{k}-\partial_{k} A_{j}
$$

where $\boldsymbol{A}=\left(A_{1}, A_{2}, \ldots, A_{n}\right)$ is an $n$-dimensional covariant function of the coordinates, $\boldsymbol{A} \equiv \boldsymbol{A}(\boldsymbol{x})$, which can be referred to as the $n$-dimensional vector potential. If $\boldsymbol{A}$ is a linear function of the coordinates then it can be expressed by an $(n \times n)$-matrix $\mathrm{A}$ (a covariant tensor) as

$$
A_{j}(x)=\mathrm{A}_{k j} x^{k}
$$

(the Einstein convention is assumed throughout). Substituting it to (1) one obtains that $H$ is simply the antisymmetrized tensor $A$, i.e.

$$
H=A-A^{T},
$$

so $\mathrm{H}$ corresponds to a constant and uniform magnetic field. Working in the radiation gauge the subsidiary condition $\nabla \cdot \boldsymbol{A}=g^{j k} \partial_{j} A_{k}=g^{j k} \mathrm{~A}_{j k}=0$ has to be assumed. In particular, this form includes the Landau gauge and the antisymmetric gauge determined by $A=H / 2$. By transposition of the matrix $A$ one obtains a magnetic field $H^{T}$ associated with $H$ :

$$
\mathrm{H}_{j k}^{\mathrm{T}}=\mathrm{A}_{j k}^{\mathrm{T}}-\mathrm{A}_{k j}^{\mathrm{T}}=-\mathrm{H}_{j k} .
$$

The Hamiltonian for a free particle in an external magnetic field, with the effective mass $m$ and the charge $q$, can be written as

$$
\mathcal{H}=\frac{1}{2 m} p^{2}=\frac{1}{2 m} g^{j k} p_{j} p_{k},
$$

with $p$ being the canonical momentum, i.e. an $n$-dimensional covariant operator

$$
p_{j}=-\mathrm{i} \hbar \partial_{j}-\frac{q}{c} \mathrm{~A}_{k j} x^{k} .
$$

These operators satisfy the commutation relation

$$
\left[p_{j}, p_{k}\right]=\mathrm{i} \hbar \frac{q}{c} \mathrm{H}_{j k},
$$

so they do not commute for $\mathrm{H}_{j k} \neq 0$. However, it is important in the further considerations that these operators are numbers, i.e. commute with any operator. Equations of motion for $p_{j}$ and $x^{k}$ are given by the commutators with the Hamiltonian

$$
\dot{p}_{j}=\frac{q}{m c} g^{k l} H_{j k} p_{l}, \quad \dot{x}^{k}=\frac{1}{m} g^{k l} p_{l} .
$$

This system of equations leads to the integrals of motion which can be determined substituting $\dot{x}$ to $\dot{p}$ [2]

$$
\frac{\mathrm{d}}{\mathrm{d} t}\left(p_{j}-\frac{q}{c} \mathrm{H}_{j k} x^{k}\right)=0 .
$$

Taking into account the definitions (1) and (5) one obtains

$$
p_{j}-\frac{q}{c} \mathrm{H}_{j k} x^{k}=-\mathrm{i} \hbar \partial_{j}-\frac{q}{c} \mathrm{~A}_{k j}^{\mathrm{T}} x^{k}=p_{j}^{\mathrm{T}} .
$$

Due to their definitions, operators $p_{j}^{\mathrm{T}}$ commute with the Hamiltonian but they do not commute with each other

$$
\left[p_{j}^{\mathrm{T}}, p_{k}^{\mathrm{T}}\right]=\mathrm{i} \hbar \frac{q}{c} \mathrm{H}_{j k}^{\mathrm{T}}=-\mathrm{i} \hbar \frac{q}{c} \mathrm{H}_{j k} .
$$


Note that for an antisymmetric gauge, $A=H / 2$, one obtains

$$
p_{j}^{\mathrm{T}}=-\mathrm{i} \hbar \partial_{j}+\frac{q}{c} \mathrm{~A}_{k j} x^{k}=-\mathrm{i} \hbar \partial_{j}+\frac{q}{c} A_{j},
$$

which agrees with the definitions of magnetic translations used by Brown and Zak $[3,4]$. The above presented form of $p_{j}^{T}$ allows us to consider any linear gauge $\boldsymbol{A}$ and to define the magnetic translations as unitary operators $[3,6,7]$

$$
T(x)=\exp \left(-\frac{\mathrm{i}}{\hbar} x^{j} p_{j}^{\mathrm{T}}\right) \text {. }
$$

\section{Cyclotronic orbits}

The definition (8) of $p_{k}^{T}$ can be written as a set of equations for variables $x^{l}$

$$
\mathrm{H}_{j k} x^{k}=\frac{c}{q}\left(p_{j}-p_{j}^{\mathrm{T}}\right) \text {. }
$$

Since $\mathrm{H}$ is an antisymmetric matrix then its eigenvalues are equal to 0 or are arranged in pairs of imaginary numbers $\pm \chi_{i} i, l=1,2, \ldots, N \leq n / 2$. Therefore, there exists such an orthogonal basis $\left\{\varepsilon_{\alpha}\right\}, \alpha=1,2, \ldots, n$, in which the magnetic tensor consists of $N$ two-dimensional antisymmetric blocks

$$
\left(\begin{array}{rr}
0 & \chi_{l} \\
-\chi_{l} & 0
\end{array}\right) \text {. }
$$

Note that this basis can be obtained by a transformation $B$ which is orthogonal with respect to a positive definite metric tensor $\gamma_{\alpha \beta}$, not with respect to a general metric tensor $g_{j k}$. It means that one can choose the basis $\left\{\boldsymbol{e}_{j}\right\}$ to be orthogonal with respect to both forms, i.e. $\left(e_{j}\right)^{l}\left(e_{k}\right)^{m} g_{l m}=g_{j k}$ and $\left(e_{j}\right)^{l}\left(e_{k}\right)^{m} \gamma_{l m}=\gamma_{j k}$ but a new basis $\varepsilon_{\alpha}=\mathrm{B}_{\alpha}^{j} e_{j}$ is orthogonal, in general, with respect to the tensor $\gamma$ only. For example, in the most interesting case when $g_{j j}=1$ for $j<n, g_{n n}=-1$ and 0 in the other cases with $\gamma_{\alpha \beta}=\delta_{\alpha \beta}$, corresponding to an $(n+1)$-dimensional space-time, the orthogonal transformation $B$ leads to a basis in which spatial and time coordinates are mixed and some basis vectors $\varepsilon_{\alpha}$ can be singular with respect to $g_{j k}$.

For the sake of clarity the coordinates of tensors $\left(\mathrm{H}_{j k}\right),\left(x^{k}\right)$ and $\left(p_{j}^{(\mathrm{T})}\right)$ in the new basis will be denoted by the Greek letters with Greek indices, i.e. $\Theta_{\alpha \beta}, \xi^{\beta}$, and $\pi_{\alpha}^{(\mathrm{T})}$, respectively. Using this notation one can obtain (to simplify notation the factor $c / q$ is included in the definition of $\pi^{(\mathrm{T})}$ )

$$
\xi^{\beta}=\mathrm{B}_{k}^{\beta} x^{k}, \quad \pi_{\alpha}^{(\mathrm{T})}=\frac{c}{q} \mathrm{~B}_{\alpha}^{j} p_{j}^{(\mathrm{T})}, \quad \Theta_{\alpha \beta}=\mathrm{B}_{\alpha}^{j} H_{j k} \mathrm{~B}_{\beta}^{k} .
$$

The $l$-th block of the matrix $\Theta_{\alpha \beta}$, with $l=1,2, \ldots, N \leq n / 2$, is given by (12), so the set of Eqs. (11) can be rewritten as

$$
\Theta_{\alpha \beta} \xi^{\beta}=\pi_{\alpha}-\pi_{\alpha}^{\mathrm{T}} \text {. }
$$

A form of the matrix $\Theta$ leads to a decomposition into two subsets of equations: for odd coordinates $\xi^{2 l-1}$ with even coordinates $\pi_{2 l}$ and vice versa

$$
\chi_{l} \xi^{2 l-1}=-\left(\pi_{2 l}-\pi_{2 l}^{\mathbf{T}}\right)
$$




$$
\chi_{l} \xi^{2 l}=\left(\pi_{2 l-1}-\pi_{2 l-1}^{\mathrm{T}}\right) .
$$

Therefore, the case $\chi_{l} \neq 0$ corresponds to a movement of a particle in the plane determined by the vectors $\xi^{2 l-1}$ and $\xi^{2 l}$. The center of the orbit is given by a pair

$$
\left(\pi_{2 l}^{\mathrm{T}},-\pi_{2 l-1}^{\mathrm{T}}\right) / \chi_{l}
$$

and the relative coordinates are

$$
\left(-\pi_{2 l}, \pi_{2 l-1}\right) / \chi_{l} \text {. }
$$

The number of independent cyclotronic movements is equal to $N \leq n / 2$, i.e. the number of non-zero values of $\chi_{l}$, and each of them is described by the Hamiltonian $\mathcal{H}_{l}=\left(\pi_{2 l-1}^{2}+\pi_{2 l}^{2}\right) / 2 m, l=1,2, \ldots, N$. Of course, it enables us to introduce the well-known and useful notation $\pi_{l}^{ \pm}=\pi_{2 l-1} \pm \mathrm{i} \pi_{2 l}$. Movements in the other directions are completely free, i.e. they are described by the Hamiltonian $\mathcal{H}_{\text {free }}=\sum_{\gamma=2 N+1}^{n}\left(\dot{\xi}^{\gamma}\right)^{2} / 2 m$. Using the determined matrix B one can express these Hamiltonians and the center coordinates in the variables $x^{k}$ and $p_{j}$. Due to commutation relations (6) and (9) each cyclotronic movement is quantized, whereas the other part of the energy spectrum is continuous.

\section{Conclusions}

It has been indicated that the movement of a charged particle in a magnetic field can always be decomposed into $N$ cyclotronic motions and $n-2 N$ free ones. This indicates the differences in the energy spectrum for $n=2$ and $n=3$ and suggests that for $n=4$ the whole energy spectrum can be discrete. However, considering the four-dimensional space-time it means that an electric field has to be included and the metric tensor of this space is not positively definite. If a magnetic field is applied only, then there is always such a basis in which the tensor $\Theta$ has only one block (12); of course this block determines a surface perpendicular to the magnetic field $\boldsymbol{H}$.

\section{Acknowledgments}

This work is partially supported by the Committee for Scientific Research within the project No. 263/P03/99/16.

\section{References}

[1] L. Landau, Z. Phys. 64, 629 (1930); R. Peierls, Z. Phys. 80, 763 (1933).

[2] M.H. Johnson, B.A. Lipmann, Phys. Rev. 76, 828 (1949).

[3] E. Brown, Phys. Rev. 133, A1038 (1964).

[4] J. Zak, Phys. Rev. 134, A1602, A1607 (1964).

[5] V.J. Menon, D.C. Agrawal, J. Phys. A, Math. Gen. 31, 1109 (1998).

[6] B. Huckestein, Rev. Mod. Phys. 67, 357 (1995).

[7] W. Florek, Acta Phys. Pol. A 92, 399 (1997). 